\title{
El Modelo living apart together (LAT) y el Modelo cohabitante en las Fuerzas Armadas españolas
}

\author{
The living apart together (LAT) model and the cohabiting \\ partner model in the Spanish Armed Forces
}

María Gómez Escarda

Universidad Rey Juan Carlos

maria.gomez@urjc.es (ESPAÑA)

Yolanda Agudo Arroyo

Universidad Nacional de Educación a Distancia (UNED)

yagudo@poli.uned.es (ESPAÑA)

Rubén J. Pérez Redondo

Universidad Rey Juan Carlos de Madrid

rubenjose.perez@urjc.es (ESPAÑA)

Recibido: 17.02 .2018

Aceptado: 22.08.2018

\section{RESUMEN}

La movilidad laboral es habitual en ciertas profesiones, entre ellas, las Fuerzas Armadas. En general, los hombres y mujeres militares han de asumir diversos cambios de destino a lo largo de sus trayectorias laborales y, en ocasiones, estos cambios llevan aparejados procesos de movilidad geográfica. Ante estas situaciones, cuando los militares tienen pareja e/o hijos pueden tomar dos alternativas. La primera es que se desplace a la nueva localidad únicamente el o la militar, dando origen a un Modelo living apart together (LAT) y, la segunda, es que la pareja o la familia al completo se trasladen también, formando parte, por tanto, de un Modelo cohabitante.

El presente artículo tiene como objetivo estudiar la presencia y características del Modelo LAT y del Modelo cohabitante en las Fuerzas Armadas españolas. Con este fin se diseñó una metodología basada en la técnica de encuesta. Se elaboró un cuestionario autoadministrado estructurado a partir de tres áreas 
temáticas (sociodemográfica, militar y de opinión) y que fue difundido por diferentes asociaciones de militares y de familias de militares españolas. Los resultados muestran cuáles son las principales variables que influyen en la decisión de optar por uno u otro modelo (laborales, económicos y familiares) y cómo repercuten ambos modelos de convivencia en la pareja y en los hijos.

\title{
PALABRAS CLAVE
}

Movilidad laboral, living apart together (LAT), pareja cohabitante, familia militar, reubicación.

\begin{abstract}
Labor mobility is common in certain professions, including the Armed Forces. Military people have to assume several changes of assignment along their carriers and, sometimes, these changes implies geographical mobility. In these situations, when the military has a partner and /or children can take two alternatives. The first is to move just the military to the new location, creating a living apart together model (LAT) and, the second, is when the couple or the whole family move together, creating a cohabiting model.

This article has the objective of studying the presence and features of the LAT Model and the Cohabiting Model in the Spanish Armed Forces. To this end, a methodology was designed based on the survey technique with a self-administred questionnaire structured from three thematic areas (sociodemographic, military and opinion) and which was spread by different associations of military and families of Spanish military.

The results show which are the main variables that affect the decision to choose one or other of two models (labor, economic and family) and how each model of living affect the couple and the children.
\end{abstract}

\section{KEY WORDS}

Labor mobility, living apart together (LAT), cohabiting partner, army family, relocation.

\section{INTRODUCCIÓN}

Los modelos de convivencia de las parejas o matrimonios en los hogares son numerosos y han sido clasificados de muy diversas maneras. La unidad de análisis en el estudio de los modelos de convivencia familiar es el hogar que se define, según el criterio de residencia, como el "grupo humano formado por una 
o varias personas que residen habitualmente en una vivienda familiar todo el año o la mayor parte del mismo, no teniendo por qué existir necesariamente vínculos familiares entre los miembros del grupo" (Instituto Nacional de EstadísticaINE). Sin embargo, en el presente trabajo este último aspecto es básico, ya que, se pretenden conocer los modelos de convivencia en los hogares entre las personas que tienen un vínculo de parentesco y, más concretamente, en el ámbito de las Fuerzas Armadas.

Menéndez (2001) establece dos modelos de convivencia familiar que recogen la pluralidad de las representaciones familiares actuales, por un lado, el modelo convencional y, por otro, el no convencional. El modelo convencional está representado por los hogares compuestos por un hombre y una mujer, con o sin descendencia, que regulan legalmente su unión, ya sea mediante el matrimonio o el registro como pareja de hecho. Por su parte, el modelo no convencional no deja de aumentar y, aunque en el pasado también se podían encontrar ejemplos no convencionales de convivencia, en muchos casos no se reconocía legalmente la igualdad de derechos de sus miembros. Los modelos no convencionales son numerosos, destacando los hogares cohabitantes y los hogares sin hijos por elección; los hogares compuestos por parejas o matrimonios del mismo sexo; aquellos en los que se producen nacimientos de madre no casada; los hogares monoparentales; los hogares compuestos por familias reconstituidas; los hogares en los que está presente el modelo "living together apart" (vivir juntos pero estar separados) o el modelo "living apart together" (vivir separados pero estar juntos).

El modelo "living together apart" comprende al menos dos adultos que mantenían anteriormente una relación de pareja y tras su ruptura continúan viviendo juntos por diversos motivos, destacando, entre otros, los económicos, el no querer separarse de sus hijos, la presión familiar, etc. (Martin, Cherlin y Cross-Barnet, 2011: 2). Por su parte, el modelo "living apart together" (LAT) es aquel en el que dos personas mantienen una relación de pareja, pero residen en viviendas separadas y basan su relación en encuentros temporales (Tai, Baxter y Hewitt, 2014: 73; Duncan y Philips, 2010: 1; Durán y Rogero, 2004: 33).

Una pareja LAT se podría definir "como alternativa a la unión conyugal, arreglos de pareja más o menos permanentes constituidos por individuos que no desean o no están disponibles por diversas razones para compartir un hogar, por lo tanto, es una relación sentimental íntima de carácter intermitente donde cada miembro de la pareja posee un domicilio propio" (Quilodrán y Hernández, 2008: 373). En opinión de Rivas (2008: 181) se termina así con la idea de que las relaciones de pareja llevan implícita la cohabitación o de que las personas que viven solas están solteras.

Este modelo de pareja se puede basar en relaciones a corta o a larga distancia y su tipología es la siguiente: en primer lugar, parejas separadas al comienzo de su relación (pre-unión); en segundo lugar, parejas separadas por razones laborales; en tercer lugar, parejas separadas por cuidados informales a personas mayores y a niños; en cuarto lugar, parejas de personas mayores; en quinto lugar, parejas que no quieren repetir errores pasados; $y$, por último, parejas separadas 
pre-ruptura (Ayuso, 2012: 590-591). La cohabitación en estas situaciones puede ser intermitente, si cada uno mantiene su vivienda y, en ocasiones uno se queda en casa del otro, o puede ser alternada, cuando la pareja es en realidad cohabitante pero, por diversos motivos, su vida trascurre entre dos viviendas (Trost y Levin, 1999; Caradec, 1996). Por tanto, una parte de las parejas LAT se forman por consenso entre los dos miembros de la misma y otras son forzadas por factores externos, como la situación familiar y las condiciones laborales (Reuschke, 2010: 218). Song-Chul (2001: 35) afirma que este tipo de convivencia es más frecuente entre los profesionales con altos ingresos que entre aquellos que se encuentran en los estratos medios o bajos. En cuanto al lugar donde residen, en general, tras haber probado al principio a vivir en alojamientos hoteleros suelen alquilar una habitación o un pequeño apartamento en el que residen durante la semana.

Las separaciones por motivos laborales parecen incrementarse por el creciente número de parejas que no quieren abandonar sus carreras profesionales (Levin, 2004: 232; Régnier-Loilier, Beaujouan y Villeneuve-Gokalp, 2009: 105; Boyle et al., 2008: 219). Sin embargo, este modelo no se da únicamente en las parejas de doble carrera, es decir, aquellas en las que ambos desarrollan un trabajo remunerado, también los estudios, las circunstancias económicas e incluso la edad de los hijos e hijas pueden intervenir, asimismo, en la decisión de que la pareja no se mude a la nueva localidad (Formankova y K囚ižkova, 2015: 108; Anderson y Spruill, 1993: 132). Generalmente, esta decisión se toma de manera consensuada en la pareja/familia y siempre teniendo en cuenta el bienestar global de todos sus miembros (McBride y Bergen, 2014: 17).

Estas parejas se convierten en parejas (o matrimonios) de fin de semana, ya que, viven separados casi toda la semana conviviendo únicamente el fin de semana y en cuanto a su tipología pueden ser, por un lado, parejas jóvenes (menos de 45 años), sin hijos y cuyo tiempo de separación se sitúa en torno al año. El origen de su separación es la oportunidad de un nuevo trabajo, en muchos casos para satisfacer la realización personal de cada uno de los miembros de la pareja. Y, por otro lado, parejas de mayor edad (más de 45 años), con hijos (Becerril, 2003; Winfield, 1985). El tiempo de separación es superior (unos 8 años) y el origen de su situación es un ascenso de alguno de los miembros de la pareja y no tanto un nuevo trabajo. Además, Song-Chul (2001:31. 35) establece una diferenciación entre las familias de fin de semana y las familias "lonely goose" que son aquellas que permanecen separadas por periodos más prolongados, conviviendo básicamente en los periodos vacacionales.

En opinión de Sahlstein (2004: 690) este modelo de convivencia tiene consecuencias negativas y positivas. Por un lado, se pueden generar conflictos entre la pareja y con los hijos, suponiendo, además, un importante desembolso económico para las familias, y repercutiendo asimismo en las oportunidades laborales de las parejas. Sin embargo, por otro lado, puede ser beneficioso al permitir, por ejemplo, una mayor libertad. Bernal (2013: 176) afirma que a pesar de no convivir de forma permanente las relaciones se basan en la solidaridad, la lealtad, el cuidado del otro y una alta valoración y admiración por su pareja si bien las

EMPIRIA. Revista de Metodología de Ciencias Sociales. N. ${ }^{\circ} 41$ septiembre-diciembre, 2018, pp. 57-78.

ISSN: 1139-5737, DOI/empiria.41.2018.22604 
responsabilidades familiares recaen principalmente sobre uno de sus miembros (Lyssens-Danneboom y Mortelmans, 2014: 963).

\section{OBJETIVOS Y METODOLOGÍA}

Debido a las especiales características de la profesión militar, principalmente la movilidad de larga duración (superior a un año), el modelo de familia LAT ha estado y continúa estando muy presente dentro de las Fuerzas Armadas españolas. Sin embargo, en España las consecuencias familiares de dicha movilidad no se han estudiado en profundidad. Con el fin de aportar datos sobre dichas consecuencias, el presente artículo tiene como objetivo estudiar la presencia, características y repercusiones del Modelo LAT (parejas que no comparten diariamente la misma residencia) y del Modelo cohabitante (parejas que comparten diariamente la misma residencia) en las Fuerzas Armadas españolas.

Para la consecución del objetivo planteado se diseñó una metodología basada en la técnica de encuesta. Para ello, inicialmente, se acotó el universo objeto de estudio a partir de los datos actualizados que facilita el Observatorio Militar para la Igualdad entre Mujeres y Hombres en las Fuerzas Armadas (2017). A fecha de diciembre de 2017, el total de efectivos en las Fuerzas Armadas españolas ascendía a 102.645 , con un $87,5 \%$ de hombres y un $12,5 \%$ de mujeres. La mayoría pertenecían al Ejército de Tierra (63,47\%), seguidos de la Armada (17,12\%), del Ejército del Aire (16,97\%) y de los Cuerpos Comunes $(2,43 \%)$. Los cuadros de mando (oficiales y suboficiales) representaban el 36,6\% del total y el resto pertenecían a tropa y marinería. A partir de estos datos se procedió a diseñar una muestra estadísticamente representativa de 450 parejas de militares $(p=q=50$ y 2 sigmas) de la que se deriva un error de $\pm 4,71 \%$.

El proceso de configuración de la muestra estaba condicionado por el hecho de que el objeto del estudio eran las parejas de los militares y no había datos oficiales sobre los que poder calcular la misma. Por esta razón se optó por intentar aproximarse, en la medida de lo posible, a una "afijación proporcional inversa" por sexo y edad (Perelló, 2009), ya que, los datos del universo son de militares y los de la muestra son de las parejas de los militares. En lo que respecta al sexo la muestra está integrada por un $65,4 \%$ de mujeres y un 35,6\% de hombres. En términos de franjas de edad, la muestra se ajusta razonablemente al perfil de edades del universo, según los datos más recientes facilitados por el Anuario Estadístico Militar (AEM, 2016) y por las Estadísticas de Personal Militar (EPM, 2016). El universo recoge un 40,7\% de personas de 33 años o menos y la muestra un $39,2 \%$. De igual modo, el universo cuenta con un 39,8\% de personas entre 34 y 45 años mientras que en la muestra esa franja asciende al 48,2\%. Por último, el porcentaje de militares de 46 y más años, suponen el $19 \%$ en el universo y el $12,4 \%$ en la muestra. Teniendo en cuenta la escala el universo está representado por un $12,6 \%$ de oficiales, un $23,4 \%$ de suboficiales y un $63,9 \%$ de militares pertenecientes a tropa o marinería. La muestra, por su parte presenta datos pa- 
rejos a estos con un $11,3 \%$ de oficiales, un $23,6 \%$ de suboficiales y un $64,9 \%$ de representantes de la escala de tropa y marinería.

A partir de la acotación de la muestra, se optó por dar forma a la encuesta a partir de un cuestionario autoadministrado ${ }^{1}$ estructurado a partir de tres áreas temáticas. En primer lugar, el área sociodemográfica que contenía variables como sexo, edad, situación familiar, situación profesional, lugar de residencia, apoyo familiar y presencia de amigos civiles y/o militares. En segundo lugar, el área propiamente militar en la que se incluía escala, ejército, lugar de destino/lugar de residencia familiar, cambios de destino, misiones/navegaciones, endogamia y apoyo institucional. Por último, se estableció un área de opinión en la que se recogía información de carácter cualitativo mediante preguntas abiertas sobre las consecuencias de la movilidad en las parejas y/o en los hijos.

\section{LA MOVILIDAD DE LARGA DURACIÓN EN LAS FUERZAS ARMADAS}

En el ámbito laboral la movilidad puede analizarse desde dos perspectivas. En primer lugar, la movilidad funcional cuando se produce un cambio de actividad dentro de la misma empresa y, en segundo lugar, la movilidad geográfica por la que los trabajadores se trasladan a un centro de trabajo dentro de la misma empresa implicando un cambio de localidad. Los frenos a la movilidad geográfica son diversos y, entre ellos, destacan los siguientes: el desarraigo cultural, familiar y social, principalmente cuando se produce un traslado de país y se desconoce el idioma de la región de destino; el posible empeoramiento de la situación laboral del cónyuge; los desajustes en los estudios de los hijos; la incertidumbre respecto al regreso; y presentar rechazo hacia otras culturas (Pin et al, 2008: 2, 13). La movilidad geográfica produce en los trabajadores ansiedad e incertidumbre, ya que, se sienten incapaces de controlar y estructurar el tiempo; además desestabiliza su equilibrio vital y les aleja de sus redes familiares y de sus amistades (Rivas, 2004: 68).

La movilidad ha sido y continúa siendo una característica fundamental de la profesión militar. Las parejas afrontan principalmente en las etapas de constitución y expansión las repercusiones negativas de la movilidad. Se producen ausencias involuntarias por parte de uno o de los dos miembros de la pareja que pueden ser la causa de conflictos familiares (Gómez, Hormigos y Pérez, 2016).

$\mathrm{Si}$ se analiza la movilidad laboral y geográfica de los distintos grupos de ocupación teniendo en cuenta el tiempo de residencia en el municipio, en el año 2017 se puede observar (tabla 1) que la mayor parte de los trabajadores llevan

${ }^{1}$ Los organismos que colaboraron en la difusión de cuestionario fueron los siguientes: Asociación Unificada de Militares Españoles (AUME); Asociación de Suboficiales de las Fuerzas Armadas (AS-FAS); Asociación de Tropa y Marinería Española (ATME); Asociación de Tropa y Marinería (AMTM); Asociación de Familias de Militares Españoles (AFME); Familia Fuerzas Armadas; Mujeres de Militares Españoles y El Rincón de las Fuerzas Armadas. 
residiendo cinco años o más en la misma localidad, sin embargo, los datos varían en las ocupaciones militares, incrementándose los porcentajes cuando la permanencia es menor (menos de un año y entre uno y menos de tres años); la diferencia no es tan significativa en el intervalo de entre tres y cinco años; y en los de mayor permanencia, los de cinco años o más, las ocupaciones militares se encuentran muy por debajo del resto, lo que demuestra que la movilidad es superior en este caso.

Tabla 1. Tiempo de residencia en el municipio en porcentaje (2017)

\begin{tabular}{lcc}
\hline & Ocupaciones militares & Total ocupaciones \\
\hline Menos de 1 año & 10,7 & 2,5 \\
Entre 1 y menos de 3 años & 12,3 & 6,5 \\
Entre 3 y menos de 5 años & 6,8 & 4,9 \\
5 o más años & 70,2 & 86,1 \\
Total & 100 & 100 \\
\hline
\end{tabular}

Fuente: Elaboración propia a partir de la Estadística de Movilidad Laboral y Geográfica, INE

Analizando únicamente los datos referidos a las ocupaciones militares la tabla 2 muestra que, efectivamente, en los últimos siete años, la mayor parte de los militares llevan cinco o más años en el mismo municipio; en segundo lugar, se encuentran los que llevan entre uno y menos de tres años, cuya tendencia en levemente decreciente desde el año 2014; en tercer lugar, los que llevan entre tres y menos de cinco años con una tendencia similar; y, por último, los que llevan menos de un año.

Tabla 2. Tiempo de residencia en el municipio en ocupaciones militares en porcentaje (2010-2017)

\begin{tabular}{ccccccccc}
\hline & 2010 & 2011 & 2012 & 2013 & 2014 & 2015 & 2016 & 2017 \\
\hline $\begin{array}{c}\text { Menos de } \\
\quad \mathbf{1} \text { año }\end{array}$ & 8,4 & 9,5 & 3,9 & 8,1 & 5,7 & 7,5 & 5,9 & 10,7 \\
$\begin{array}{c}\text { Entre 1 y } \\
\text { menos de }\end{array}$ & 11,4 & 11,4 & 18,2 & 14,2 & 21,8 & 13,3 & 14,9 & 12,3 \\
$\quad 3$ años & & & & & & & & \\
$\begin{array}{c}\text { Entre 3 y } \\
\text { menos de }\end{array}$ & 8,5 & 11,6 & 13,6 & 9,8 & 9,2 & 12,5 & 8,1 & 6,8 \\
$\quad 5$ años \\
$\begin{array}{c}5 \text { o más } \\
\text { años }\end{array}$ & 71,7 & 67,5 & 64,3 & 67,9 & 63,3 & 66,7 & 71,1 & 70,2 \\
$\quad$ Total & 100 & 100 & 100 & 100 & 100 & 100 & 100 & 100 \\
\hline
\end{tabular}

Fuente: Elaboración propia a partir de la Estadística de Movilidad Laboral y Geográfica, INE 
Dentro de la movilidad geográfica en las Fuerzas Armadas españolas es necesario diferenciar si ésta se produce de manera voluntaria o forzosa y si la movilidad es de corta o de larga duración.

La movilidad es voluntaria cuando es el militar el que decide libremente cambiar de destino o realizar algún tipo de formación fuera de su lugar de residencia. Por el contrario, la movilidad es forzosa cuando de forma involuntaria el militar se ve obligado a cambiar de destino por ascenso, por servidumbre tras la realización de algún curso (Curso de Mando de Unidades Paracaidistas, Diploma de Operaciones Especiales, etc.) o por rebasar el límite temporal de seis meses pendiente de asignación de destino como ocurre, por ejemplo, en la reincorporación del personal una vez finalizada una Comisión de Servicio en el extranjero.

Entre las medidas de conciliación de la vida familiar y profesional en las Fuerzas Armadas se establece que no podrán ser destinados con carácter forzoso a una provincia diferente los matrimonios o parejas de hecho duales (ambos militares) que tengan hijos menores de doce años a su cargo y que estén destinados en la misma provincia, excepto en aquellas vacantes correspondientes a jefes de unidad, centro u organismo,; las que se ofertan a quien se incorpora a una escala o supera determinados cursos de perfeccionamiento o de altos estudios de la defensa nacional; ni al personal en la situación de servicio activo pendiente de asignación de destino (R.D. 577/2017). Además, en el caso de las parejas o matrimonios duales cuando uno de los miembros de la pareja hubiese sido destinado con carácter forzoso y/o voluntario a otra provincia el otro miembro de la pareja queda exento del plazo de mínima permanencia para poder solicitar vacante a la misma provincia que su cónyuge o pareja de hecho, si no está ocupando un destino de jefe de unidad, centro u organismo y siempre que se acceda desde otra provincia distinta a la solicitada (Guía medidas de conciliación-FAS 2018: 18-19).

En la tabla 3 se muestran los datos de movilidad forzosa de los militares españoles (Ejército de Tierra, Armada y Ejército del Aire) por escalas en el año 2014. Teniendo en cuenta los porcentajes destaca que los oficiales $(6,91 \%)$ se encuentran muy por encima de la media total de movilidad (4,77\%). Una posible explicación es el tiempo de permanencia por empleos, ya que, en el caso de los oficiales los ascensos se producen, en general, cada menos tiempo, lo que conlleva cambio de destino por cambio de empleo. Lo contrario ocurre en el caso de los suboficiales $(3,98 \%)$, ya que, generalmente se publican vacantes que cubren varios empleos, por ejemplo, Sargento/Sargento Primero o Brigada/Subteniente, por lo que se supone que tienen mayor permanencia en sus destinos. La tropa y marinería se encuentra cercana a la media global $(4,69 \%)$ debido a que cada cambio de empleo supone, en general, un cambio de destino con carácter forzoso. 
Tabla 3. Destino de carácter forzoso por escalas (2014)

\begin{tabular}{lccc}
\hline & Efectivos & Forzosos & Porcentaje \\
\hline Oficiales & 14.132 & 976 & 6,91 \\
Suboficiales & 27.500 & 1.079 & 3,98 \\
Tropa o Marinería & 75.915 & 3.557 & 4,69 \\
Total & 117.547 & 5.612 & 4,77 \\
\hline
\end{tabular}

Fuente: Memoria Informe 2014 (Observatorio de la Vida Militar)

La movilidad de larga duración es aquella superior a un año y que puede ser ocasionada por los diferentes periodos formativos y los cambios de destino (dentro o fuera de España) que pueden llevar aparejada una reubicación. Por su parte, la movilidad de corta duración sería aquella inferior a un año y que puede derivarse de los despliegues en misiones internacionales y las maniobras y navegaciones. En este caso el análisis se va a centrar en la movilidad de larga duración.

En el caso de las Fuerzas Armadas españolas los principales inconvenientes que presenta la movilidad geográfica son la vivienda, la escolarización de los hijos y el trabajo del cónyuge, siendo estos los principales motivos por los que la familia no se traslada a la nueva localidad de destino (Observatorio de la Vida Militar, 2014, 15).

Cuando la familia se enfrenta a un proceso de reubicación se plantean, generalmente, dos situaciones: la primera, es que sea el o la militar la que se desplace permaneciendo el resto en la residencia familiar y dando origen, por tanto, a un Modelo LAT de convivencia y, la segunda, es que toda la familia se traslade al nuevo destino, principalmente si dicha reubicación se va a prolongar en el tiempo, dando lugar a un Modelo cohabitante. Se tome la opción que se tome las repercusiones para la familia son numerosas, tanto para la persona que debe desplazarse, como para sus hijos y su pareja.

En lo que respecta a la primera opción, es decir, que la familia permanezca en la residencia habitual dando origen a un Modelo LAT, se produce principalmente en el caso de que las parejas posean un empleo y no quieran o no puedan renunciar a él. La influencia de los hijos también es clave, ya que, la decisión de acompañar o no al militar depende de variables como la edad que tengan, los estudios que estén realizando e, incluso, su situación personal y/o afectiva. Destacan asimismo el entorno familiar, ya que, en esta decisión puede influir el hecho de tener algún familiar enfermo y/o dependiente, y, también, los problemas de vivienda (Gómez, 2013). En el Modelo LAT, la red solidaria principal y básica es la familiar y la ayuda y presencia de los progenitores es esencial (Becerril, 2003). Con ellos se tiene un contacto frecuente y son las personas que más apoyo pueden ofrecer, dependiendo, evidentemente, de la distancia a la que residan. La segunda red de apoyo estaría configurada por los amigos, si bien, parece que en estas familias se produce un alejamiento debido a la falta de tiempo. En último lugar, se encontraría el apoyo institucional. 
En cuanto a la segunda opción, es decir, el Modelo cohabitante, en el pasado, lo habitual era que la familia del militar se trasladara en cada cambio de destino, debido principalmente a que la pareja no solía desempeñar un trabajo remunerado. En la actualidad la pareja sigue renunciando, en muchas ocasiones, a su formación y a su estabilidad laboral, sobre todo, si se tienen hijos pequeños y no se posee un empleo remunerado. En estas situaciones lo habitual es que se traslade toda la familia siendo las mujeres jóvenes las que en mayor medida sufren esta situación, mientras que según se va incrementando la edad aumenta la probabilidad de que permanezcan en la misma localidad durante un periodo de tiempo prolongado y puedan encontrar y mantener un empleo, e incluso iniciar y/o terminar sus estudios (Harrell et al., 2004: 20).

La movilidad afecta a las oportunidades laborales y formativas de las parejas de los militares, presentando como principales barreras tener una baja cualificación, lo que puede convertirse en la mayor traba para obtener un empleo; la movilidad, que supone un conflicto entre el ámbito familiar y laboral, ya que, la pareja se encuentra en la tesitura de abandonar su trabajo por su familia o de continuar en él separando, entonces, a su familia; el cuidado de los descendientes; y las actitudes de los empleadores que no quieren a personas que no vayan a permanecer durante un tiempo prolongado en puestos de trabajo (Centre for Social Justice, 2016; De Angelis y Weschler, 2015: 33; Cooney, De Angelis y Wechsler, 2011: 363).

En otras ocasiones el problema no es tener una baja cualificación sino, más bien, lo contrario, ya que, un gran porcentaje de candidatos están sobre cualificados con respecto a las ofertas de trabajo a las que han decidido optar (Pérez, 2014: 32). Asimismo, hay que añadir que, otro de los principales problemas que deben afrontar las parejas que tenían un empleo y renuncian a éste para trasladarse, es que no tienen la posibilidad de recibir la prestación por desempleo, ya que, si el cese en la empresa es voluntario no están en situación legal de desempleo y, en consecuencia, no cumplen uno de los requisitos para acceder dicha prestación (González, Miller y Trail, 2016: 23).

Además de los problemas laborales, estos cambios de residencia provocan el alejamiento de su núcleo familiar original y de sus amistades y, para los hijos cambios de colegios y de amigos (Adao, 2010: 128). Algunos autores defienden la idea de esta situación produce alienación. Las parejas y los hijos de los militares sienten un sentimiento de desarraigo cuando se trasladan a una nueva localidad, en la que carecen del apoyo de su entorno más cercano (Burrell, 2006: 48). Croan, LeVine y Blankinship (1992: viii) afirman que los problemas en los procesos de reubicación son tres veces mayores en el caso de los militares con pareja e hijos, sobre todo, en lo relativo a cuestiones logísticas, y en las familias con mayor número de hijos y, principalmente, cuando son mayores de tres años, ya que pueden presentar dificultades de adaptación más elevadas. 


\section{RESULTADOS: EL MODELO LAT Y EL MODELO COHABITANTE EN LAS FUERZAS ARMADAS ESPAÑOLAS}

A continuación, se van a mostrar los resultados obtenidos en el cuestionario analizando, en primer lugar, los datos generales de la muestra relacionados con la movilidad de larga duración y, en segundo lugar, los datos relativos a la presencia del Modelo LAT o del Modelo cohabitante en la muestra.

Analizando los datos generales y centrando el análisis en los cambios de destino que llevan aparejada una reubicación, dos tercios de los encuestados afirman que sus parejas han tenido que afrontar a lo largo de sus carreras algún cambio de destino que llevara aparejado un cambio de residencia. El 33\% han cambiado una o dos veces, el $16 \%$ tres, el $8,5 \%$ cuatro y el resto cinco o más.

En la Armada y los Cuerpos Comunes más del $80 \%$ de las repuestas indican que han tenido que afrontar un proceso de reubicación, en el Ejército de Tierra el $63,8 \%$ y en el Ejército del Aire el 59,7\%. Por escalas, la respuesta es positiva en el $80 \%$ de los oficiales y de los suboficiales y en el 59,6\% de la tropa y marinería. En cuanto a las ocasiones en las que se han producido estos cambios, de las 290 respuestas obtenidas (tabla 4), los oficiales son los que mayor movilidad han afrontado, debido a que, como se ha apuntado, el tiempo de permanencia en el empleo es menor, seguidos de los suboficiales y, por último, la tropa y marinería. Estos datos pueden estar relacionados con el hecho de que, la tropa y marinería elige su primer destino de forma voluntaria al ingresar en las Fuerzas Armadas $\mathrm{y}$, su tiempo de permanencia en los destinos y en los empleos, es mayor que en los casos anteriores, de hecho, no suelen promocionar hasta tener asegurada una vacante en el destino que desean.

Tabla 4. Cambios de destino con cambios de residencia por escala

\begin{tabular}{|c|c|c|c|}
\hline & Oficial & Suboficial & Tropa y marinería \\
\hline \multirow{2}{*}{$1 \mathrm{vez}$} & 8 & 21 & 67 \\
\hline & $20,0 \%$ & $25,3 \%$ & $40,1 \%$ \\
\hline \multirow{2}{*}{2 veces } & 13 & 27 & 57 \\
\hline & $32,50 \%$ & $32,50 \%$ & $34,1 \%$ \\
\hline \multirow{2}{*}{3 veces } & 5 & 17 & 25 \\
\hline & $12,5 \%$ & $20,5 \%$ & $15,0 \%$ \\
\hline \multirow{2}{*}{4 veces } & 4 & 10 & 9 \\
\hline & $10,0 \%$ & $12,0 \%$ & $5,4 \%$ \\
\hline \multirow{2}{*}{5 o más veces } & 10 & 8 & 9 \\
\hline & $25,0 \%$ & $9,6 \%$ & $5,4 \%$ \\
\hline \multirow{2}{*}{ Total } & 40 & 83 & 167 \\
\hline & $100 \%$ & $100 \%$ & $100 \%$ \\
\hline
\end{tabular}


Los datos muestran que en estos procesos de reubicación las parejas no siempre han acompañado a los militares. La variable sexo muestra pocas diferencias, el 55,7\% de hombres no han acompañado a sus parejas frente a un 57,8\% de mujeres que sí lo han hecho. Por edades son las parejas más jóvenes y las más mayores las que en mayor medida han acompañado a sus parejas, mientras que más de la mitad de los intervalos intermedios, es decir, de 31 a 40 años y de 41 a 50 años, afirman no haber acompañado a sus parejas a la nueva localidad. En los intervalos intermedios la situación laboral suele ser más estable y los hijos presentan edades más elevadas, por lo que es más probable que la familia no se desplace. Precisamente, la tabla 5 muestra si la pareja ha acompañado al militar en la reubicación teniendo en cuenta su situación laboral.

Tabla 5. Movilidad de la pareja ante una reubicación según su situación laboral

\begin{tabular}{lrr}
\hline & No & Sí \\
\hline Estudiante & 7 & 11 \\
Jubilado/a o pensionista (antes ha trabajado) & $4,9 \%$ & $6,8 \%$ \\
& 1 & 0 \\
Otra situación & $0,7 \%$ & $0,0 \%$ \\
& 2 & 1 \\
Parado/a (buscando trabajo) & $1,4 \%$ & $0,6 \%$ \\
Pensionista (antes no ha trabajado) & 17 & 40 \\
Tiene un trabajo remunerado & $11,9 \%$ & $24,7 \%$ \\
& 0 & 1 \\
Trabajo doméstico no remunerado & $0,0 \%$ & $0,6 \%$ \\
& 111 & 83 \\
Total & $77,6 \%$ & $51,2 \%$ \\
& 5 & 26 \\
\end{tabular}

Fuente: Elaboración propia

$*[\otimes 2(6, N=305)=29,702, \mathrm{p}<0,001]$

Los datos recogidos de las 305 respuestas muestran que, efectivamente, la situación laboral es una variable fundamental, ya que, el hecho de estar desempeñando un trabajo remunerado influye en la decisión de no acompañar a la pareja, mientras que, si se está en paro, si la pareja se dedica al trabajo doméstico no remunerado o si es estudiante es más probable que acompañe a su pareja. También puede ocurrir que la reubicación haya provocado un empeoramiento de su situación laboral, encontrándose un mayor porcentaje en paro, pero buscando trabajo y dedicados al trabajo doméstico no remunerado y un menor porcentaje de personas que están trabajando. 
Tras este análisis general, se va a exponer a continuación los resultados referidos a la parte de la muestra en la que las provincias de destino y de residencia no coindicen, teniendo en cuenta si han acompañado o no a su pareja en el proceso de reubicación. El Modelo LAT sería aquel en el que no se ha acompañado a la pareja y el Modelo cohabitante sería aquel en el que la pareja o toda la familia han acompañado al militar en el proceso de reubicación.

Del total de la muestra analizada, un tercio $(33,3 \%)$ de los encuestados han respondido que sus parejas trabajan en una provincia diferente de aquella en la que se ubica su residencia familiar. Si bien hay que apuntar que no en todos los casos una respuesta negativa en esta pregunta indica que las parejas sean LAT, debido a que en ocasiones se producen movimientos pendulares, es decir, los militares se trasladan diariamente a la provincia de trabajo y regresan a pernoctar a su residencia familiar. En la tabla 6 se observa que el Modelo LAT está representado por el 68,5\% de los 308 encuestados que han respondido a la pregunta, que afirman que la provincia de destino y de residencia de su pareja no coinciden y que no les han acompañado en dicha reubicación. El Modelo cohabitante está representado, por tanto, por el 31,5\% de las personas desplazadas que sí que les han acompañado y cohabitan con sus parejas.

Tabla 6. Cambio de destino/cambio de residencia

\begin{tabular}{|c|c|c|c|c|}
\hline & \multicolumn{2}{|c|}{ ¿Ha acompañado a su pareja? } & \multirow{2}{*}{ Total } \\
\hline & & No & Sí & \\
\hline \multirow{6}{*}{$\begin{array}{l}\text { Provincia de destino/ } \\
\text { provincia de residen- } \\
\text { cia }\end{array}$} & \multirow{2}{*}{ No } & 89 & 41 & 130 \\
\hline & & $68,5 \%$ & $31,5 \%$ & $100 \%$ \\
\hline & \multirow[t]{2}{*}{ Sí } & 66 & 112 & 178 \\
\hline & & $37,1 \%$ & $62,9 \%$ & $100 \%$ \\
\hline & \multirow[t]{2}{*}{ Total } & 155 & 153 & 308 \\
\hline & & $50,3 \%$ & $49,7 \%$ & $100 \%$ \\
\hline
\end{tabular}

Fuente: Elaboración propia

$*[\otimes 2(1, \mathrm{~N}=308)=29,599, \mathrm{p}<0,001]$

En general en ambos modelos los militares del Ejército de Tierra son los más representados, seguidos del Ejército del Aire y la Armada con porcentajes similares y, por último, los Cuerpos Comunes. Por escala también en ambos la mayor representación se da en los soldados y marineros, seguida de los suboficiales (con un valor algo más elevado en el Modelo cohabitante) y, en último lugar los oficiales. Las diferencias en cuanto al sexo no son reseñables, ya que, en ambos modelos la presencia es algo mayor en el caso de las mujeres.

Analizando la edad de las personas encuestadas se puede afirmar que, en ambos modelos, el intervalo más representado está entre 31 y 40 años, seguido 
de aquellas que tienen 30 años o menos, las que tienen de 41 a 50 años y una representación residual de mayores de 50 años. En ambos modelos la situación familiar es muy parecida, la mayor parte están casados/as, en segundo lugar, están los solteros/as con valores más altos en el Modelo LAT (30,3\%) que en el Modelo cohabitante (19,5\%), en tercer lugar, las parejas de hecho y, por último, los separados/as o divorciado/as.

Según los datos obtenidos las variables que más influyen a la hora de decantarse por uno u otro modelo son el hecho de tener o no un empleo $(43,3 \%)$, los motivos económicos (mantenerse con un sueldo, pagar una hipoteca, etc.) $(18,9 \%)$, los motivos familiares (principalmente por los hijos y por no querer alejarse del resto de familiares) $(18,1 \%)$, el ser una pareja dual (ambos militares) con destinos diferentes $(11,8 \%)$ y los estudios $(7,8 \%)$.

Si se estudia la situación laboral de las parejas, se puede observar que no varía mucho en los dos modelos establecidos, ya que, la gran mayoría desempeñan un trabajo remunerado, aunque en el Modelo LAT el valor asciende al 67,4\% de los encuestados y en el Modelo cohabitante al 61\%, siendo la mayoría asalariados/as. El porcentaje de parados/as buscando trabajo es más elevado en el Modelo cohabitante, con el 22\% de los encuestados frente al 16,9\% en el Modelo LAT. Y lo mismo ocurre en el caso de las personas dedicadas al trabajo doméstico no remunerado, en el primer caso con un 12,2\% y en el segundo con un 5,6\%. El resto de opciones de respuesta (jubilados/as, pensionistas y otras situaciones) presentan valores mínimos o no han sido seleccionadas por los encuestados.

Como se ha expuesto, el hecho de tener un empleo remunerado puede influir en la decisión de no acompañar a la pareja en el cambio de destino y, una reubicación puede influir, también, en las posibilidades de la pareja de encontrar y mantener un empleo. Las respuestas cualitativas así lo recogen:

Tener que dejar mi trabajo anterior y después de estar buscando trabajo en la provincia de destino de mi pareja, trabajar a 60 kilómetros de mi lugar de residencia (encuestada/o 95).

La repercusión negativa principal es tener que «renunciar» a mi vida profesional, ya que es muy difícil tener que empezar de cero cada vez que hay un cambio de destino (encuestada/o 112).

Llevo nueve meses sin encontrar trabajo (encuestada/o 114).

Abandonar un puesto de trabajo con futuro, dificultades para encontrar otro por no conocer el sitio y gente (encuestada/o 120).

$Y$ si me traslado con él, como he hecho en esta ocasión, debo tener paciencia y empezar de cero a buscar trabajo (encuestada/o 168).

Tuve que renunciar a un puesto de responsabilidad con buen sueldo y posición (encuestada/o 183).

Debido a su cambio de destino, dejé mi empleo con contrato fijo en una gran empresa multinacional (encuestada/o 193).

He tenido que abandonar mi trabajo en dos ocasiones (encuestada/o 219).

En cuanto a si el trabajo que desempeñan se corresponde con su formación, se puede observar (tabla 7) que de los 126 encuestados que forman la submuestra analizada, en el caso de las parejas LAT más de la mitad afirman que su trabajo 
se corresponde con su formación, mientras que en el caso de las parejas convivientes el 65,9\% afirman que su trabajo no se corresponde con su formación.

Tabla 7. ¿Se corresponde su trabajo con su formación?

\begin{tabular}{crrrr}
\hline & \multicolumn{1}{c}{ Sí } & \multicolumn{1}{c}{ No } & No sabe & \multicolumn{1}{c}{ Total } \\
\hline Modelo LAT & 47 & 36 & 2 & 85 \\
& $55,3 \%$ & $42,4 \%$ & $2,3 \%$ & $100 \%$ \\
Modelo Cohabitante & 14 & 27 & 0 & 41 \\
& $34,1 \%$ & $65,9 \%$ & $0,0 \%$ & $100 \%$ \\
\hline Fuente: Elaboración propia & & & & \\
$*[\square 2(2, \mathrm{~N}=126)=6,575, \mathrm{p}<0,005]$ & & & &
\end{tabular}

En el Modelo LAT es más probable que se pueda encontrar y mantener un empleo acorde con la formación mientras que en el Modelo cohabitante, son numerosas las parejas que renuncian a sus empleos por la reubicación familiar. Cuando llegan a la nueva localidad se enfrentan a dificultades para encontrar un empleo y más, si se pretende que sea acorde a la formación, teniendo que aceptar, en mayor medida, empleos de cualificación inferior. Esta situación se recoge asimismo en algunas de las respuestas recogidas en el cuestionario:

Dificultad para mantener un trabajo estable y acorde con mi formación profesional (encuestada/o 102).

Tuve que trabajar en cosas que no tienen nada que ver con mi formación (encuestada/o 117).

En lo que respecta a los principales motivos por los que las parejas no desempeñan un trabajo remunerado, en el Modelo LAT el 60\% afirman que el principal motivo es la dificultad para conciliar la vida familiar y/o laboral, el 32\% por no encontrar trabajo y el $8 \%$ por elección personal. Como se ha expuesto en este Modelo las responsabilidades familiares recaen en exclusiva en uno de los miembros de la pareja, por lo que, los problemas para conciliar se incrementan. Mientras que en Modelo cohabitante el 50\% afirman que el principal motivo es no encontrar trabajo, el $43,8 \%$ por dificultades para conciliar y el $6,2 \%$ por elección personal.

En lo que respecta a los hijos en el Modelo LAT más de la mitad de los encuestados no tienen (52,8\%) mientras que en el Modelo cohabitante más de la mitad afirma que sí los tiene (53,7\%). Teniendo en cuenta el número de hijos no existen diferencias significativas entre ambos grupos, la mayor parte tienen dos hijos, seguidos de los que tienen uno/a, y, por último, los que tienen tres hijos. En cuanto a su edad en ambos modelos los mayores porcentajes se encuentran en los de seis años o menos y en los de siete a doce años. Sin embargo, las diferencias son importantes cuando se analiza quién se hace cargo habitualmente de ellos. En general, tanto en el Modelo LAT como en el Modelo cohabitante son 
las parejas de los militares las que, en mayor medida, se hacen cargo de los hijos, siendo la segunda opción más elegida "ambos conjuntamente" con un porcentaje más elevado, como es evidente, en el segundo modelo.

Teniendo en cuenta las repuestas cualitativas obtenidas las repercusiones en los hijos son algo diferentes en ambos modelos. En el Modelo LAT la convivencia se reduce prácticamente al fin de semana y, no en todos los casos, ya que, por las características del destino y/o por la distancia geográfica que separa a la pareja, se pueden reducir las posibilidades de desplazamiento,

El padre de mis hijos está en Madrid y nosotros en Málaga, viene los fines de semana llegando viernes por la noche y volviéndose el domingo al mediodía (si no hay servicio de ningún tipo, claro está) (encuestada/o 116).

Están lejos de su padre ya que solo lo ven los fines de semana (encuestada/o 379).

Los principales problemas se plantean por la manera en que los hijos reaccionan ante la separación de uno o de ambos progenitores,

Cambios en actitud por falta del padre (encuestada/o 44).

A la niña le afectó psicológicamente el separarse de su madre (encuestada/o 60).

Mis hijos lo pasan mal, lloran y lo echan de menos, más aún cuando alguna que otra semana ni tan siquiera pueden verlo (encuestada/o 116).

Mi hijo el mayor se descentra mucho cuando su padre no está. Y mi hija pequeña, desde que nació, su padre en sus cinco primeros años de vida no estaba prácticamente nunca, o bien cambio de destino o misiones fuera. Hasta tal punto llegó ese desapego que no lo daba nunca besos, es como si la figura paterna en todo ese tiempo no hubiera existido (encuestada/o 195).

En el Modelo cohabitante los principales problemas están relacionados con su reacción ante la separación de la familia y los amigos y su adaptación al nuevo entorno. A estas situaciones hay que añadir que, en numerosas ocasiones, los traslados se producen durante el curso escolar por lo que las repercusiones negativas se multiplican. En general las respuestas muestran que las dificultades son numerosas incrementándose con la edad y, que, en casos extremos se producen problemas de índole psicológica y fracaso escolar,

Notas más bajas, le cuesta más hacer amigos y acostumbrarse a su nuevo entorno (encuestada/o 68).

La adaptación a los nuevos colegios representa una dificultad mayor cuanto mayor son los hijos (encuestada/o 71).

Mis hijos han sido unos inadaptados, por perder tantos amigos y sentirse sin unas raíces, por culpa de los cambios de colegios, no han llegado a adaptarse y la pequeña ha sido la que más lo ha sufrido, ha tenido fracaso escolar (encuestada/o 219).

Las dificultades para conciliar en el Modelo LAT son importantes, ya que, no se puede contar con el apoyo de la pareja y el peso de las responsabilidades familiares recaen sobre el miembro de la pareja que se queda en el domicilio familiar. En estas situaciones el apoyo familiar es fundamental, aunque, también lo 
es en las parejas que son reubicadas fuera de la localidad de residencia familiar, ya que, si no pueden contar con el apoyo de la familia, o este es intermitente, pueden tener más dificultades para encontrar y/o mantener un empleo. Precisamente, la tabla 8 muestra que, en el caso de las 130 respuestas de la submuestra, en el Modelo LAT el 62,9\% de los encuestados afirman que sus familias residen en su misma provincia frente a un 73,2\% de los representantes del Modelo cohabitante que afirman, lo contrario, es decir, que su familia no reside en la misma localidad.

Tabla 8. ¿Reside parte de su familia en la misma provincia que usted?

\begin{tabular}{cccc}
\hline & Sí & No & Total \\
\hline Modelo LAT & 56 & 33 & 89 \\
& $62,9 \%$ & $37,1 \%$ & $100 \%$ \\
Modelo Cohabitante & 11 & 30 & 41 \\
& $26,8 \%$ & $73,2 \%$ & $100 \%$ \\
\hline
\end{tabular}

Fuente: Elaboración propia*

$[\square 2(1, \mathrm{~N}=130)=14,639, \mathrm{p}<0,001]$

Precisamente una de las principales repercusiones negativas planteadas por los encuestados en el Modelo cohabitante es la separación de la familia y los amigos,

Un inconveniente ha sido incrementar la distancia con miembros de la familia para que puedan ayudarnos con el hijo (encuestada/o 5).

Distancia de la familia/amigos, pérdida del círculo de apoyo, aislamiento (encuestada/o 26).

Abandonar mi lugar de residencia con el correspondiente alejamiento de amigos y familiares (encuestada/o 105).

La negativa es que me tenido que alejar de mi familia y ahora estamos solos aquí sin ayuda (encuestada/o 126).

Imposibilidad de encontrar trabajo por no tener apoyo familiar (encuestada/o 176).

La segunda red de apoyo son los amigos en el Modelo LAT el 66,3\% de los encuestados afirman tener más amigos civiles frente al 36,6\% en el Modelo cohabitante, en este caso, la respuesta mayoritaria $(41,5 \%)$ ha sido que tienen más o menos el mismo número de amigos civiles que militares o relacionados con el ámbito militar, destacando, asimismo, el 22\% que afirma que tienen más amigos militares. Ante una reubicación las familias han de comenzar de nuevo en la localidad de destino, en muchos casos, la residencia se encuentra cercana al cuartel y el contacto más frecuente se produce con personas o familias que están viviendo o han vivido la misma situación, creando vínculos y redes de solidaridad y apoyo mutuo, 
Lo único que la familia está lejos, pero aquí los amigos ayudan (encuestada/o 222).

Hacer buenos amigos (encuestada/o 404).

El apoyo institucional, por su parte, se centra principalmente en el pago de la mudanza, en ayudas económicas por desplazamientos y en descuentos en transportes. Sin embargo, estas ayudas se consideran, en general, escasas,

Les aportan información de trámites de papeleos, pero no les ayudan ni a buscar piso ni nada simplemente les proporcionan una ayuda económica para el alquiler proporcional a como estén los alquileres en la ciudad de destino, pero en la mayoría de aspectos es empezar de cero (encuestada/o 211).

Una compensación por la vivienda, que dura 36 meses y no da para pagar ni un alquiler (encuestada/o 353).

Relacionado con el apoyo institucional, los problemas económicos son, como se ha expuesto, otra de las razones que pueden influir a la hora de tomar la decisión de que la familia permanezca en el hogar o se desplace a la nueva localidad. Estas dificultades suelen estar relacionadas con la vivienda y el coste que suponen los desplazamientos semanales,

No tenemos medios económicos para hacer frente a tener otra residencia (encuestada/o 125).

Gastos dobles en vivienda (encuestada/o 273).

Por el coste de billetes ya que vivimos en Canarias y él está destinado en la Península (encuestada/o 262).

Por no poder afrontar el alquiler (encuestada/o 391).

A pesar de que las repercusiones de los modelos analizados y, principalmente del Modelo cohabitante, son generalmente negativas, los encuestados han destacado también algunos aspectos positivos en ambos modelos. En el Modelo LAT el hecho de que la pareja no renuncie a sus aspiraciones profesionales y a la estabilidad de sus hijos favorece la estabilidad familiar. En el Modelo cohabitante se destaca, especialmente, la posibilidad de mantener una convivencia diaria con su pareja e hijos, conocer a otras personas y lugares diferentes,

Lo positivo es que trabaja en la misma ciudad y lo vemos más que en el antiguo destino (encuestada/o 126).

Conocer lugares nuevos para mi familia, hacer nuevos contactos (encuestada/o 172).

Poder estar al lado de mi marido (encuestada/o 192).

\section{CONCLUSIONES Y DISCUSIÓN}

La movilidad geográfica ha sido y continúa siendo una característica básica de la profesión militar. A la hora de analizar la movilidad en el ámbito militar se puede diferenciar entre la movilidad voluntaria o forzosa y la movilidad de corta (menos de un año) o de larga duración (más de un año). La movilidad de larga duración, en la que se centra la investigación, está relacionada con los cambios 
de destino o los periodos formativos y, en el caso de las Fuerzas Armadas españolas, los principales inconvenientes que presenta son la vivienda, la escolarización de los hijos y el trabajo del cónyuge.

Analizando los datos obtenidos en el cuestionario autoadministrado se observa que dos tercios de los encuestados afirman que sus parejas han tenido que afrontar algún cambio de destino que llevara aparejado un cambio de residencia. En lo que respecta a los ejércitos, han tenido que afrontar este proceso más del $80 \%$ en la Armada y los Cuerpos Comunes, el $63,8 \%$ en el Ejército de Tierra y el $59,7 \%$ en el Ejército del Aire. Por escalas, el $80 \%$ de los oficiales y suboficiales y el $59,6 \%$ de la tropa y marinería han vivido esta situación.

Las repercusiones de la movilidad de larga duración sobre las parejas/familias de los militares son diversas. La principal es que dicha movilidad puede transformar los modelos de convivencia dependiendo de si, en el proceso de reubicación, se desplaza a la nueva localidad sólo el o la militar, dando origen a un Modelo living apart together (LAT), o si lo hace también la pareja o la familia al completo, formando parte, por tanto, de un Modelo cohabitante. La decisión de optar por uno u otro modelo depende de diversas variables, entre las que destacan la situación laboral y la situación familiar.

En lo que respecta a la situación laboral puede influir en la decisión de no acompañar a la pareja. En ambos modelos la mayoría de las parejas tienen un trabajo remunerado, aunque en el caso del Modelo LAT el porcentaje es algo mayor. Por su parte, las cifras de personas en paro buscando trabajo y dedicadas al trabajo doméstico no remunerado son más elevadas en el Modelo cohabitante. En este caso, muchas parejas renuncian a sus carreras profesionales y se enfrentan a dificultades para encontrar un empleo en la nueva localidad y, más aún, si buscan un empleo acorde a su formación.

La situación familiar también ejerce una enorme influencia. En el Modelo LAT las dificultades para conciliar son importantes, ya que, las responsabilidades familiares recaen, principalmente, sobre uno de los miembros de la pareja y, además, los hijos presentan dificultades por vivir separados de uno de sus progenitores. En el Modelo cohabitante los principales problemas están relacionados con su reacción ante la separación de la familia y los amigos y su adaptación al entorno escolar. Familia y amigos son los principales apoyos en los dos modelos y el apoyo institucional es, en general, catalogado como insuficiente.

El presente artículo supone el inicio de una línea de trabajo novedosa en España y en la que se pretenden conocer las características sociodemográficas y laborales de las parejas de los y las militares y las repercusiones de la movilidad en las familias. Asimismo, se ha dado la oportunidad a las personas encuestadas de mostrar los que consideran que son los puntos fuertes y puntos débiles en la relación entre la profesión militar y la familia y de establecer propuestas de mejora que pueden resultar muy útiles a la hora de conocer las necesidades y opiniones de las familias del personal militar. 


\section{REFERENCIAS}

ADAO, M. C. (2010): "Aspects of adhesion of women to the military values: marriage and military family", História (online), 29(2), pp.116-134

ANDERSON, L. A. y SPRUILL, J. W. (1993): "The dual-career commuter family: A lifestyle on the move", Marriage \&Family Review,19, pp.131-147

AYUSO, L. (2012): "Living apart together en España. ¿Noviazgos o parejas independientes?", RIS, 3(7), pp. 587-613

BECERRIL, D. (2003): "Cuando el trabajo separa: los matrimonios de fin de semana", Revista Portularia 3, pp. 179-206

BERNAL, I. C. (2013): "Juntos aunque separados. Parejas LAT en la ciudad de Medellín", Revista de la Facultad de Trabajo Social, 29(29), pp. 173-194

BOYLE, P. J. et al. (2008): "Moving and union dissolution", Demography, 1(45), pp. 209-222

BURRELL, L. (2006): "Moving military families: the impact of relocation on family well-being, employment and commitment to the military" en Military life. The Psichology of serving in peace and combat the military family, 3, Wesport,Greenwood Publishing Group, pp. 39-63

CARADEC, V. (1996): “Les formes de la vie conjugale des 'jeunes' couple 'agés”, Population, 51, pp. 897-928

CASTRO, T. y DOMÍNGUEZ, M. (2008): "Matrimonios "sin papeles": Perfil sociodemográfico de las parejas de hecho en España según el Censo de 2001", Política y Sociedad, 2, pp. 51-72

CENTRE FOR SOCIAL, JUSTICE (2016): Military Families and Transition, Reino Unido, Centre for Social Justice

COONEY, R., DE ANGELIS K. y WECHSLER, M. (2011): "Moving with the Military: Race, Class, and Gender Differences in the Employment Consequences of Tied Migration," Race, Gender \& Class, 18 (1/2), pp. 360-384

CROAN, G. M., LEVINE, C. T. y BLANKINSHIP, D. A. (1992): Family Adjustment to Relocation, Technical Report 968, U.S. Army Research Institute for the Behavioral and Social Sciences

DE ANGELIS, K. y WESCHLER, M. (2015): “Transitions in the military and the family as greedy institutions" en Military Families and War in the 21st Century, Londres, Routledge, pp. 22-42

DUNCAN, S., y PHILIPS, M. (2010): "People who live apart together (LATs): How different are they?", Sociological Review, 58(1), pp. 112-134

DURÁN, M. A. y ROGERO, J. (2004): "Nuevas parejas para viejas desigualdades". Revista de Estudios de Juventud, 67, pp. 25-37

FLAQUER, LL. (1999): La estrella menguante del padre. Barcelona, Editorial Ariel

FORMANKOVA, L. y K囚IŽKOVA, A. (2015): "Love Will Keep Us Apart? Understanding Living Apart Together Partnerships in the Post-state-socialist Czech Republic", Sociologicky 冈asopis/Czech Sociological Review, 51 (6), pp. 993-1022

GÓMEZ, M. (2013): La familia en las Fuerzas Armadas españolas, Ministerio de Defensa

GÓMEZ, M., HORMIGOS, J. y PÉREZ, R. J. (2016): “Familia y suelo pegajoso en las fuerzas armadas españolas”, Revista mexicana de sociología, 78(2), pp. 203-228

GONZÁLEZ, G. C., MILLER, L. L. y TRAIL, T. E. (2016): The Military Spouse Education and Career Opportunities. Program Recommendations for an Internal Monitoring System, Santa Mónica, RAND Corporation 
TAI, T., BAXTER, J. y HEWITT, B. (2014): “Do co-residence and intentions make a difference? Relationship satisfaction in married, cohabiting, and living apart together couples in four countries", Demographic Research; 31 (3), pp. 71-104

LEVIN, I. (2004): "Living apart together: A new family form”, Current Sociology 52(2), pp. $223-240$

LYSSENS-DANNEBOOM, V. y MORTELMANS, D. (2014): "Living Apart Together and Money: New Partnerships, Traditional Gender Roles", Journal of Marriage and Family, 76, pp. 949-966

MARTIN, C., CHERLIN, A., y CROSS-BARNET, C. (2011): "Living Together Apart in France and the United States", Population, 66(3-4), pp. 561-581

MCBRIDE, M. C. y BERGEN, K. M. (2014): "Voices of women in commuter marriages: A site of discursive struggle", Journal of Social and Personal Relationships, 4 (31), pp. 554-572

MENÉNDEZ, S. (2001): "La diversidad familiar en España. Un análisis de su evolución reciente y su aceptación", Apuntes de Psicología, 3, pp. 367-386

MINISTERIO DE DEFENSA (2011): Real Decreto 456/2011, de 1 de abril, por el que se aprueba el Reglamento de destinos del personal militar profesional, BOE, $\mathrm{N}^{\mathrm{o}} 81$, de 5 de abril, pp. 34.835-34.851

MINISTERIO DE DEFENSA (2017): Real Decreto 577/2017, de 12 de junio, por el que se modifica el Reglamento de destinos del personal militar profesional aprobado por el Real Decreto 456/2011, de 1 de abril, BOE, $N^{\circ} 140$, de 13 de junio, pp. 48.54248.549

MORA, G. (2001): "Las familias españolas antes el cambio de siglo" en Estructura y cambio social, Madrid, CIS, pp. 293-312

OBSERVATORIO MILITAR PARA LA IGUALDAD ENTRE MUJERES Y HOMBRES EN LAS FUERZAS ARMADAS (2015): Memoria Informe año 2014, disponible en http://www.defensa.gob.es/ministerio/organigrama/subdef/digenper/omi/ [consulta: 15/02/2018]

OBSERVATORIO MILITAR PARA LA IGUALDAD ENTRE MUJERES Y HOMBRES EN LAS FUERZAS ARMADAS (2017): Informe 2017 de evolución de la mujer y el hombre en las Fuerzas Armadas, disponible en http://www.defensa.gob. es/ministerio/organigrama/subdef/digenper/omi/ [consulta: 15/02/2018]

OBSERVATORIO MILITAR PARA LA IGUALDAD ENTRE MUJERES Y HOMBRES EN LAS FUERZAS ARMADAS (2018): Guía para la aplicación de las medidas de conciliación en las Fuerzas Armadas, disponible en http://www.defensa. gob.es/ministerio/organigrama/subdef/digenper/omi/ [consulta: 15/02/2018]

PERELLÓ, S. (2009): Metodología de la investigación social, Madrid, Dykinson.

PÉREZ, R. J. (2014): "Una relación desequilibrada: la selección de personal en tiempos de crisis", methaodos. revista de ciencias sociales, 2(1), pp. 23-35

PIN, J. R. et al. (2008): La movilidad geográfica en la empresa: un análisis para España, Estudio $\mathrm{N}^{\circ}$ 60, IESE-Universidad de Navarra

QUILODRÁN, J. y HERNÁNDEZ, R. (2008): "Vivir juntos, aunque separados (LAT): el surgimiento de una nueva modalidad de convivencia conyugal" en Familias iberoamericanas ayer y hoy. Una mirada interdisciplinaria, México, ALAP Editor, pp. 369-394

RÉGNIER-LOILIER, A., BEAUJOUAN, E. y VILLENEUVE-GOKALP, C. (2009): "Neither single, nor in a couple: A study of living apart together in France", Demographic Research, 21 (4), pp. 75-108 
REUSCHKE, D. (2010): "Living apart together over long distance-time-space patterns and consequences of a late-modern living arrangement", Erdkunde, 64(3), pp. 215226

RIVAS, A. M. (2004): "Del trabajo como valor de inscripción social al trabajo como factor de desestructuración social: el caso de los trabajadores y trabajadoras desplazados por la deslocalización industrial dentro del estado español", Cuadernos de Relaciones Laborales, 2(22), pp. 63-82

RIVAS, A. M. (2008): "Las nuevas formas de vivir en familia: el caso de las familias reconstituidas", Cuadernos de Relaciones Laborales, 1(26), pp. 179-202.

SAHLSTEIN, E. M. (2004): "Relating at a distance: Negotiating being together and being apart in long-distance relationships", Journal of Social and Personal Relationships, 21(5), pp. 689-710

KIM, S. C. (2001): “Weekend Couples' amongst Korean Professionals: an ethnography of living apart on weekdays", Korea Journal,41(4): 28-47.

TROST, J. y LEVIN, I. (1999): "Parejas sin domesticidad común”, Desacatos. Revista de Ciencias Sociales 2

WINFIELD, E. (1985): Commuter marriage: Living together, apart, Nueva York, Colombia University Press 